\title{
Lupus nephritis in males; updates to current knowledge
}

\author{
Faizan Shaukat $^{1}$, Liliya Russinova $^{2}$, Bagzhan Seizhanova $^{2}$, Assel Rakhmetova $^{* * \mathbb{1}}$ \\ ${ }^{1}$ Department of Medicine, Shifa College of Medicine, Islamabad, Pakistan \\ ${ }^{2}$ Department of Internal Medicine, Kazakh National Medical University, Almaty, Kazakhstan \\ ${ }^{3}$ Department of Laboratory Medicine and Pathology, University of Alberta Hospital, Edmonton, Canada
}

\section{A R T I C L E I N F O}

Article Type:

Mini Review

\section{Article History:}

Received: 10 May 2018

Accepted: 7 December 2018

ePublished: 6 January 2019

\section{Keywords:}

Systemic lupus erythematosus

Lupus nephritis

Males

Renal outcome

End stage renal disease

\begin{abstract}
A B S T R A C T
Studies have demonstrated that male systemic lupus erythematosus (SLE) patients present with atypical manifestations, serious kidney involvement and worse prognosis. This article summarizes the findings obtained from the literature review of 16 studies to obtain an understanding of the gender correlation with SLE and lupus nephritis. We compared the influence of gender on various factors of SLE like incidence of lupus nephritis, age at manifestation of SLE, time between SLE manifestation and lupus nephritis diagnosis, frequency of dialysis, mortality risk and biopsy findings. Studies included in our review composed of remote published work, case-control studies, case reports and cross-sectional studies. Of 16 studies, 14 were conducted exclusively on lupus nephritis in SLE with comparison of gender correlation and remaining 2 studied SLE generally without any gender comparison. Six studies pointed to an increase in incidence of lupus nephritis in males, 9 studies demonstrated no disparity in gender, and one study showed contradicting results. In addition, 4 studies pointed that males had a more severe renal outcome as revealed by laboratory tests. Interestingly the age at which SLE manifests was the same in both genders according to 2 studies. Moreover, the frequency of dialysis and remission were similar between both genders. There is no consensus if these atypical manifestations are more common in males. Although some studies do show a significant increase in lupus nephritis among male SLE patients, others showed no difference or contradicting results. Hence, we suggest further studies comparing the disease manifestations.
\end{abstract}

Implication for health policy/practice/research/medical education:

Although some studies showed a significant increase in lupus nephritis among male systemic lupus erythematosus patients, others showed no difference or contradicting results. Hence, we suggest further studies comparing the disease manifestations between the two genders.

Please cite this paper as: Shaukat F, Russinova L, Seizhanova B, Rakhmetova A. Lupus nephritis in males; updates to current knowledge. J Nephropharmacol. 2019;8(1):e09. DOI: 10.15171/npj.2019.09

\section{Introduction}

Systemic lupus erythematosus (SLE) is a chronic multisystem autoimmune disorder with a circulation of autoantibodies and deposition of immune complexes in various organs and systems. SLE is known for heterogenicity of clinico-pathological manifestations and life-threatening outcomes. According to the American society of rheumatology SLE classification criteria, the diagnosis is based on the distinctive clinical features and presence of auto-antibodies. The disease etiology remains unknown, however, numerous risk factors are associated with the onset and progression of SLE, such as ethnicity, age and gender (1). SLE tends to affect young woman of child-bearing age and the incidence rate of SLE is 6-10 times more prevalent in women than in men (2). One of the most important poor prognostic factor associated with an increased rate of mortality is the development of lupus nephritis. Time from the disease onset and establishing a clinical diagnosis of lupus nephritis was described to be more prolonged in males due to overlooking of SLE symptoms (3). More random presentation of SLE in males leads to the less awareness and underestimating of SLE symptoms in male patients group. Moreover, morphological presentation and its progression to the end stage of renal disease among male patients was described as more severe rather than female counterparts (2-5). However, opinions of the male gender as a risk factor for lupus nephritis varies. The onset of lupus nephritis, and 
progression and also long-term prognosis in males is disputed in the literature.

\section{Objectives}

The main gain of this literature review is to distinguish a difference in respect to clinical presentation, morphological class with activity index, and renal outcome prognosis in male patients with lupus nephritis. We sought to put a clear line if male gender is a risk factor for an increased chance of developing lupus nephritis in SLE patients through a critical review of the literature.

\section{Materials and Methods for preclinical investigations}

For this mini-review, we used different sources, such as PubMed, Scopus, Google Scholar, Uptodate. The following keywords were used; lupus nephritis in males, systemic erythematosus lupus nephritis gender differences, male predominance in autoimmune diseases, lupus nephritis prognosis in males.

\section{Results}

Among the 16 articles, 6 showed increased incidence of lupus nephritis in males. Nephritis was the second most common manifestation of SLE in men (40.9\%) (6). SLE affects kidneys in males more often than the female cohorts (1,4,7-9,). Males with SLE regardless of ethnicity tend to have more renal involvement than females (10). In males, the spectrum of clinical presentation primarily involves infections, cardiovascular and renal diseases (11). One study suggested contrasting result: Increase of renal insufficiency among women rather than male patients (6.8\% versus $4.4 \%$ respectively) (12). Additionally one study revealed no gender disparities in lupus nephritis patients (11).

Two independent investigations showed that no gender difference exists in the mean age at the time of diagnosis $(4,12)$. One report suggested that the time from SLE diagnosis to the manifestation of lupus nephritis was decreased in males (13). Another group of researchers revealed a contradicting result and proved that this time was rather increased in males (3). Regarding disease onset, four studies demonstrated a more serious manifestation of renal disease in males, evidenced by acute kidney injury, oliguria, and anuria, nephrotic syndrome (a large amount of proteinuria, decreased serum albumin levels) and increase in serum creatinine value (5,13-15). Similarly, there are four articles showing that biopsy findings of class IV diffuse lupus nephritis was more prevalent in males (25) and only one case report describing classes III and V of lupus nephritis (16). In contrast, there was only one expert group indicating that no such difference existed between genders (14). Furthermore, morphological activity grade and indices of lupus nephritis were found to be more severe in male than in female groups $(3,13,14)$. Two studies pointed that men had a higher rate of flare $(80 \%$ of men versus $60 \%$ of women) and recurrent flare frequency compared to females (44\% versus $18.2 \%$ respectively) (2). Similarly, occurrence of relapse was higher in males than in female patients (37.5\% versus $11.4 \%$ correspondingly) (3). One study showed that males had a decreased frequency of complete remission (36.6\% versus 50.4\%) (13). Conversely one study reported the absence of distinction in remission rate between two cohorts (5). One study revealed that males tend to require dialysis more than females (4). However another authors showed no differences of males over females were found in the frequency of receiving dialysis and renal transplantation (14). Finally, three studies reported that the mortality rate was higher among males than females $(3,11,15)$. However, three other articles showed that there is no genderassociated death due to lupus nephritis in males $(5,13,14)$.

\section{Discussion}

SLE is a severe autoimmune disease affecting multiple organs and systems. It is a well- known fact, that SLE usually develops in young females rather than males (1). In males, the spectrum of clinical presentation primarily involves infections, cardiovascular and renal diseases; whereas, women tend to have mucocutaneous and musculoskeletal manifestations $(1,4,7-9,11)$. There has been increased debate about the incidence of lupus nephritis and its gender correlation. According to a population study in Slovak, nephritis was the second most common presentation of SLE in men (40.9\%) (6). Independent comparative studies by Andrade et al (7) and Hwang et al (4), emphasized that SLE affects the kidneys in males more often than the female cohorts, that data was supported by an earlier literature review by Lu et al (9). In contrast to the studies described above, a 30-year work completed by Renau AI et al, showed an increase of renal insufficiency among women rather than male patients (6.8\% versus $4.4 \%$ respectively) (12). However, conflicting results were obtained very recently by Falasinnu et al, wherein no gender disparities were found in patients with lupus nephritis (11). Surprisingly varied outcomes corresponding to ethnic backgrounds and genders were found in SLE patients from different research groups, even when controlling for geographical distribution. Nevertheless, males with SLE regardless of ethnicity tend to have more renal involvement than females (10). Thus, the influence of sex on the prevalence of lupus nephritis among males remains undetermined.

Another important question to address is the age at which SLE manifests, as it predominantly affects women during their reproductive years. Likewise, according to some reports males are more vulnerable to SLE in their thirties, which shows that no gender difference exists in the mean age at the time of diagnosis $(4,12)$. A retrospective Chinese study revealed contrary findings to the generally accepted opinions, regarding the time of disease onset and establishing lupus nephritis diagnosis. They found that the time from disease manifestation and lupus nephritis 
diagnosis was reduced in males (13). This contradicts a previous report by Wang et al, who discovered that the time from disease onset to establishing a diagnosis was prolonged in males compared to females (10.2 months versus 14.4 months). This was attributed to subtle disease presentation or the underdiagnosing of lupus nephritis symptoms in the male population (3).

With regards to clinical and laboratory findings, males diagnosed with lupus nephritis are more predisposed to acute kidney injury, oliguria, and anuria, nephrotic syndrome (a large amount of proteinuria, decreased serum albumin levels) and increased serum creatinine values (5,13-15).

Furthermore, renal biopsies provide critical information in assessing lupus nephritis in males with SLE. The revised 2003 ISN/RPS classification provides essential information based on the six-principal classes of lupus nephritis, disease activity and chronicity, tubular and vascular damage. Class IV diffuse lupus nephritis, which is generally associated with poor renal survival, is more prevalent and is the predominant class of lupus nephritis in males (2-5), rather than randomly reported classes III and V (16). Yet, this data was in contrast with an earlier report by de Carvalho et al, where no difference in histopathological class was noted between genders (14). Furthermore, morphological activity grade, which is usually determined based on endocapillary hypercellularity, and chronicity indices of lupus nephritis was reported to be more severe in male than in female groups $(3,13,14)$. Men had a higher rate of flares ( $80 \%$ of men versus $60 \%$ of women) and frequency of recurrent flares compared to females (44\% versus $18.2 \%$ respectively) (2). Similar results were obtained in an 8-year study by Wang et al that included 315 consecutive patients out of which 45 were males. Wang et al also found that the occurrence of relapse was higher in males than in female patients (37.5\% versus $11.4 \%$ correspondingly) (3). A 2018 comprehensive survey of gender impact on longterm renal outcome revealed that males had a decreased frequency of complete remission (36.6 versus 50.4\%) (13). Conversely, a retrospective joint analysis of male and female patients with SLE from Spain and Uruguay hospitals did not find a difference in remission rates between genders (5).

Male gender as a risk factor for receiving kidney replacement therapy and mortality rate due to lupus nephritis remains questionable. While one research group has documented that no differences of males over females were found in the frequency of receiving dialysis and renal transplantation (14) others claim that males tend to receive dialysis more often than females (4).

The mortality rate was two to six times higher among males than females $(3,15)$ and the average age of mortality was found to be 54 for women and 63 for men patients (11). Nevertheless, there are proponents suggesting that there is no gender-associated death due to lupus nephritis in males. $(5,13,14)$.

\section{Conclusion}

There is no consensus on the debate of male sex being a risk factor for increased incidence of lupus nephritis, poorer renal outcomes and increased rate of mortality due to lupus nephritis. We suggest that multicenter study including different ethnicities, age, risk factors for mortality, and the genetically background is necessary to gain clarity regarding the role of male gender in lupus nephritis.

\section{Authors' contribution}

FSh, LR, BS, and AR conducted the research. AR and FSh designed and supervised the study, analyzed the data and prepared the final draft of the article. All authors read and signed the final paper.

\section{Conflict of interest}

The authors declare that they have no conflict of interest.

\section{Ethical considerations}

Ethical issues (including plagiarism, data fabrication, double publication) have been completely observed by the authors.

\section{Funding/Support}

None.

\section{References}

1. Almaani S, Meara A, Rovin. B.H. Update on Lupus Nephritis. Clin J Am Soc Nephrol. 2017; 12: 825-835.

2. Resende AL, Titan SM, Barros RT, Woronik V. Worse renal outcome of lupus nephritis in male patients: a case-control study. Lupus. 2011; 20:561-7.

3. Wang YF, Xu YX, Tan Y, Yu F, Zhao MH. Clinicopathological characteristics and outcomes of male lupus nephritis in China. Lupus. 2012; 21:1472-81.

4. Hwang J, Lee J, Ahn JK, Park EJ, Cha HS, Koh EM. Clinical characteristics of male and female Korean patients with systemic lupus erythematosus: a comparative study. Korean J Intern Med. 2015; 30:242-9.

5. Urrestarazú A, Otatti G, Silvariño R, Garau M, Coitiño R, Alvarez A, et al. Lupus nephritis in males: clinical features, course, and prognostic factors for end-stage renal disease. Kidney Int Rep. 2017 2:905-912.

6. Blažíčková S, Rovenský J. Systemic lupus erythematosus detected in males in the population of Slovakia. Adv kidney Dis Treat. 2017; 1:1.

7. Andrade RM, Alarcón GS, Fernández M, Apte M, Vilá LM, et al. Accelerated damage accrual among men with systemic lupus erythematosus. Arthritis Rheum. 2007; 56: 622-30.

8. Agrawaal KK, Dhakal SS. Systemic lupus erythematosus in males: a case series. Saudi J Kidney Dis Transpl. 2014; 25:638-42.

9. Lu LJ, Wallace DJ, Ishimori ML, Scofield RH, Weisman MH. Weisman. Male systemic lupus erythematosus: a review of sex disparities in this disease. Lupus. 2010; 19:119-29. doi: 10.1177/0961203309350755.

10. Murphy G, Isenberg D. Effect of gender on clinical 
presentation in systemic lupus erythematosus. Rheumatology (Oxford). 2013; 52:2108-15.

11. Falasinnu T, Chaichian Y, Simard JF. Impact of Sex on Systemic Lupus Erythematosus-Related Causes of Premature Mortality in the United States. J Womens Health (Larchmt). 2017; 26:1214-21.

12. Renau AI, Isenberg DA. Male versus female lupus: a comparison of ethnicity, clinical features, serology and out come over a 30 year period. Lupus. 2012;21:1041-8.

13. Wei Peng, Yi Tang, · Li Tan, Wei Qin. Clinicopathological study of male and female patients with lupus nephritis: a retrospective study. Int Urol Nephrol.2018; 50:313-20.
14. de Carvalho JF, do Nascimento AP, Testagrossa LA, Barros RT, Bonfá E. Male gender results in more severe lupus nephritis. Rheumatol Int. 2010; 30:1311-5.

15. Xiong L, Tian N, Hong L, Zhang W, Chen W, Yu X. Clinicopathological Characteristics and Outcomes in Male Patients with Lupus Nephritis. Hong Kong J Nephrol. 2015; $17: 2$.

16. Miller-Hodges E, Dominiczak AF, Jennings GL, Oparil S, Batlle DC, Elijovich F, et al. Hypertension and Its Complications in a Young Man with Autoimmune Disease. Hypertension. 2017;69:536-44.

Copyright $\odot 2019$ The Author(s); Published by Society of Diabetic Nephropathy Prevention. This is an open-access article distributed under the terms of the Creative Commons Attribution License (http://creativecommons.org/licenses/by/4.0), which permits unrestricted use, distribution, and reproduction in any medium, provided the original work is properly cited. 